\title{
Partial Randomness and Dimension of Recursively Enumerable Reals
}

\author{
Kohtaro Tadaki \\ Research and Development Initiative, Chuo University \\ JST, CREST \\ 1-13-27 Kasuga, Bunkyo-ku, Tokyo 112-8551, Japan \\ E-mail: tadaki@kc.chuo-u.ac.jp
}

\begin{abstract}
A real $\alpha$ is called recursively enumerable ("r.e." for short) if there exists a computable, increasing sequence of rationals which converges to $\alpha$. It is known that the randomness of an r.e. real $\alpha$ can be characterized in various ways using each of the notions; program-size complexity, Martin-Löf test, Chaitin $\Omega$ number, the domination and $\Omega$ likeness of $\alpha$, the universality of a computable, increasing sequence of rationals which converges to $\alpha$, and universal probability. In this paper, we generalize these characterizations of randomness over the notion of partial randomness by parameterizing each of the notions above by a real $T \in(0,1]$, where the notion of partial randomness is a stronger representation of the compression rate by means of program-size complexity. As a result, we present ten equivalent characterizations of the partial randomness of an r.e. real. The resultant characterizations of partial randomness are powerful and have many important applications. One of them is to present equivalent characterizations of the dimension of an individual r.e. real. The equivalence between the notion of Hausdorff dimension and compression rate by program-size complexity (or partial randomness) has been established at present by a series of works of many researchers over the last two decades. We present ten equivalent characterizations of the dimension of an individual r.e. real.
\end{abstract}

Key words: algorithmic randomness, recursively enumerable real, partial randomness, dimension, Chaitin $\Omega$ number, program-size complexity, universal probability

\section{Introduction}

A real $\alpha$ is called recursively enumerable ("r.e." for short) if there exists a computable, increasing sequence of rationals which converges to $\alpha$. The randomness of an r.e. real $\alpha$ can be characterized in various ways using each of the notions; program-size complexity, Martin-Löf test, Chaitin $\Omega$ number, the domination and $\Omega$-likeness of $\alpha$, the universality of a computable, increasing sequence of rationals which converges to $\alpha$, and universal probability. These equivalent characterizations of randomness of an r.e. real are summarized in Theorem 6 (see Section 3), where the equivalences are established by a series of works of MartinLöf 9], Schnorr [14, Chaitin [4, Solovay [15, Calude, Hertling, Khoussainov and 
Wang 1], Kučera and Slaman [7, and Tadaki [20, between 1966 and 2006. In this paper, we generalize these characterizations of randomness over the notion of partial randomness, which was introduced by Tadaki [18 19] and is a stronger representation of the compression rate by means of program-size complexity. We introduce many characterizations of partial randomness for an r.e. real by parameterizing each of the notions above on randomness by a real $T \in(0,1]$. In particular, we introduce the notion of $T$-convergence for a computable, increasing sequence of rationals and then introduce the same notion for an r.e. real. The notion of $T$-convergence plays a crucial role in these our characterizations of partial randomness. We then prove the equivalence of all these characterizations of partial randomness in Theorem 8, our main result, in Section 4.

On the other hand, by a series of works of Ryabko 1213, Staiger 1617, Tadaki [1819], Lutz [8, and Mayordomo [10] over the last two decades, the equivalence between the notion of compression rate by program-size complexity (or partial randomness) and Hausdorff dimension seems to be established at present. The subject of the equivalence seems to be one of the most active areas of the recent research of algorithmic randomness. In the context of the subject, we can consider the notion of the dimension of an individual real in particular, and this notion plays a crucial role in the subject. As one of the main applications of our main result on partial randomness, i.e., Theorem 8 , we can present many equivalent characterizations of the dimension of an individual r.e. real.

The paper is organized as follows. We begin in Section 2 with some preliminaries to algorithmic information theory and partial randomness. In Section 3 , we review the previous results on the equivalent characterizations of randomness of an r.e. real. Our main result on partial randomness of an r.e. real is presented in Section 4. In Section 5 we apply our main result on partial randomness to give many equivalent characterizations of the dimension of an r.e. real. In Section 6 , we investigate further properties of the notion of $T$-convergence, which plays a crucial role in our characterizations of the partial randomness and dimension of r.e. reals. We conclude this paper with a mention of the future direction of this work in Section 7 Due to the 12-page limit, we omit most proofs. A full paper which describes all the proofs and other related results is in preparation.

\section{Preliminaries}

We start with some notation about numbers and strings which will be used in this paper. $\mathbb{N}=\{0,1,2,3, \ldots\}$ is the set of natural numbers, and $\mathbb{N}^{+}$is the set of positive integers. $\mathbb{Q}$ is the set of rational numbers, and $\mathbb{R}$ is the set of real numbers. A sequence $\left\{a_{n}\right\}_{n \in \mathbb{N}}$ of numbers (rationals or reals) is called increasing if $a_{n+1}>a_{n}$ for all $n \in \mathbb{N}$.

$\{0,1\}^{*}=\{\lambda, 0,1,00,01,10,11,000, \ldots\}$ is the set of finite binary strings, where $\lambda$ denotes the empty string. For any $s \in\{0,1\}^{*},|s|$ is the length of $s$. A subset $S$ of $\{0,1\}^{*}$ is called prefix-free if no string in $S$ is a prefix of another string in $S$. For any partial function $f$, the domain of definition of $f$ is denoted by $\operatorname{dom} f$. We write "r.e." instead of "recursively enumerable." 
Normally, $o(n)$ denotes any function $f: \mathbb{N}^{+} \rightarrow \mathbb{R}$ such that $\lim _{n \rightarrow \infty} f(n) / n=$ 0 . On the other hand, $O(1)$ denotes any function $g: \mathbb{N}^{+} \rightarrow \mathbb{R}$ such that there is $C \in \mathbb{R}$ with the property that $|g(n)| \leq C$ for all $n \in \mathbb{N}^{+}$.

Let $\alpha$ be an arbitrary real. For any $n \in \mathbb{N}^{+}$, we denote by $\left.\alpha\right|_{n} \in\{0,1\}^{*}$ the first $n$ bits of the base-two expansion of $\alpha-\lfloor\alpha\rfloor$ with infinitely many zeros, where $\lfloor\alpha\rfloor$ is the greatest integer less than or equal to $\alpha$. Thus, in particular, if $\alpha \in[0,1)$, then $\left.\alpha\right|_{n}$ denotes the first $n$ bits of the base-two expansion of $\alpha$ with infinitely many zeros. For example, in the case of $\alpha=5 / 8,\left.\alpha\right|_{6}=101000$.

A real $\alpha$ is called r.e. if there exists a computable, increasing sequence of rationals which converges to $\alpha$. An r.e. real is also called a left-computable real. Let $\alpha$ and $\beta$ be arbitrary r.e. reals. Then $\alpha+\beta$ is r.e. If $\alpha$ and $\beta$ are non-negative, then $\alpha \beta$ is r.e. On the other hand, a real $\alpha$ is called right-computable if $-\alpha$ is left-computable. We say that a real $\alpha$ is computable if there exists a computable sequence $\left\{a_{n}\right\}_{n \in \mathbb{N}}$ of rationals such that $\left|\alpha-a_{n}\right|<2^{-n}$ for all $n \in \mathbb{N}$. It is then easy to see that, for every $\alpha \in \mathbb{R}, \alpha$ is computable if and only if $\alpha$ is both left-computable and right-computable. A sequence $\left\{a_{n}\right\}_{n \in \mathbb{N}}$ of reals is called computable if there exists a total recursive function $f: \mathbb{N} \times \mathbb{N} \rightarrow \mathbb{Q}$ such that $\left|a_{n}-f(n, m)\right|<2^{-m}$ for all $n, m \in \mathbb{N}$. See e.g. Weihrauch 23 for the detail of the treatment of the computability of reals and sequences of reals.

\subsection{Algorithmic information theory}

In the following we concisely review some definitions and results of algorithmic information theory [45]. A computer is a partial recursive function $C:\{0,1\}^{*} \rightarrow$ $\{0,1\}^{*}$ such that $\operatorname{dom} C$ is a prefix-free set. For each computer $C$ and each $s \in\{0,1\}^{*}, H_{C}(s)$ is defined as $\min \left\{|p| \mid p \in\{0,1\}^{*} \& C(p)=s\right\}$ (may be $\infty$ ). A computer $U$ is said to be optimal if for each computer $C$ there exists $d \in \mathbb{N}$ with the following property; if $p \in \operatorname{dom} C$, then there is $q \in \operatorname{dom} U$ for which $U(q)=C(p)$ and $|q| \leq|p|+d$. It is easy to see that there exists an optimal computer. We choose a particular optimal computer $U$ as the standard one for use, and define $H(s)$ as $H_{U}(s)$, which is referred to as the program-size complexity of $s$ or the Kolmogorov complexity of $s$. It follows that for every computer $C$ there exists $d \in \mathbb{N}$ such that, for every $s \in\{0,1\}^{*}, H(s) \leq H_{C}(s)+d$.

For any optimal computer $V$, Chaitin's halting probability $\Omega_{V}$ of $V$ is defined as $\sum_{p \in \operatorname{dom} V} 2^{-|p|}$. The real $\Omega_{V}$ is also called Chaitin $\Omega$ number.

Definition 1 (weak Chaitin randomness, Chaitin [4]5]). For any $\alpha \in \mathbb{R}$, we say that $\alpha$ is weakly Chaitin random if there exists $c \in \mathbb{N}$ such that $n-c \leq$ $H\left(\alpha \uparrow_{n}\right)$ for all $n \in \mathbb{N}^{+}$.

Chaitin [4] showed that, for every optimal computer $V, \Omega_{V}$ is weakly Chaitin random.

Definition 2 (Martin-Löf randomness, Martin-Löf [9]). A subset $\mathcal{C}$ of $\mathbb{N}^{+} \times\{0,1\}^{*}$ is called a Martin-Löf test if $\mathcal{C}$ is an r.e. set and

$$
\forall n \in \mathbb{N}^{+} \quad \sum_{s \in \mathcal{C}_{n}} 2^{-|s|} \leq 2^{-n}
$$


where $\mathcal{C}_{n}=\{s \mid(n, s) \in \mathcal{C}\}$. For any $\alpha \in \mathbb{R}$, we say that $\alpha$ is Martin-Löf random if for every Martin-Löf test $\mathcal{C}$, there exists $n \in \mathbb{N}^{+}$such that, for every $k \in \mathbb{N}^{+}$, $\left.\alpha\right|_{k} \notin \mathcal{C}_{n}$.

Theorem 1 (Schnorr [14]). For every $\alpha \in \mathbb{R}, \alpha$ is weakly Chaitin random if and only if $\alpha$ is Martin-Löf random.

The program-size complexity $H(s)$ is originally defined using the concept of program-size, as stated above. However, it is possible to define $H(s)$ without referring to such a concept, i.e., as in the following, we first introduce a universal probability $m$, and then define $H(s)$ as $-\log _{2} m(s)$. A universal probability is defined as follows 24 .

Definition 3 (universal probability). A function $r:\{0,1\}^{*} \rightarrow[0,1]$ is called a lower-computable semi-measure if $\sum_{s \in\{0,1\}^{*}} r(s) \leq 1$ and the set $\{(a, s) \in$ $\left.\mathbb{Q} \times\{0,1\}^{*} \mid a<r(s)\right\}$ is r.e. We say that a lower-computable semi-measure $m$ is a universal probability if for every lower-computable semi-measure $r$, there exists $c \in \mathbb{N}^{+}$such that, for all $s \in\{0,1\}^{*}, r(s) \leq c m(s)$.

The following theorem can be then shown (see e.g. Theorem 3.4 of Chaitin [4] for its proof).

Theorem 2. For every optimal computer $V$, the function $2^{-H_{V}(s)}$ of $s$ is a universal probability.

By Theorem 2, we see that $H(s)=-\log _{2} m(s)+O(1)$ for every universal probability $m$. Thus it is possible to define $H(s)$ as $-\log _{2} m(s)$ with a particular universal probability $m$ instead of as $H_{U}(s)$. Note that the difference up to an additive constant is nonessential to algorithmic information theory.

\subsection{Partial randomness}

In the works [1819, we generalized the notion of the randomness of a real so that the degree of the randomness, which is often referred to as the partial randomness recently [2]11]3], can be characterized by a real $T$ with $0<T \leq 1$ as follows.

Definition 4 (weak Chaitin $\boldsymbol{T}$-randomness). Let $T \in \mathbb{R}$ with $T \geq 0$. For any $\alpha \in \mathbb{R}$, we say that $\alpha$ is weakly Chaitin $T$-random if there exists $c \in \mathbb{N}$ such that $T n-c \leq H\left(\left.\alpha\right|_{n}\right)$ for all $n \in \mathbb{N}^{+}$.

Definition 5 (Martin-Löf $\boldsymbol{T}$-randomness). Let $T \in \mathbb{R}$ with $T \geq 0$. A subset $\mathcal{C}$ of $\mathbb{N}^{+} \times\{0,1\}^{*}$ is called a Martin-Löf T-test if $\mathcal{C}$ is an r.e. set and

$$
\forall n \in \mathbb{N}^{+} \quad \sum_{s \in \mathcal{C}_{n}} 2^{-T|s|} \leq 2^{-n}
$$

For any $\alpha \in \mathbb{R}$, we say that $\alpha$ is Martin-Löf T-random if for every Martin-Löf $T$-test $\mathcal{C}$, there exists $n \in \mathbb{N}^{+}$such that, for every $\left.k \in \mathbb{N}^{+}, \alpha\right\rceil_{k} \notin \mathcal{C}_{n}$. 
In the case where $T=1$, the weak Chaitin $T$-randomness and Martin-Löf $T$-randomness result in weak Chaitin randomness and Martin-Löf randomness, respectively. Tadaki [19] generalized Theorem 1 over the notion of $T$-randomness as follows.

Theorem 3 (Tadaki [19]). Let $T$ be a computable real with $T \geq 0$. Then, for every $\alpha \in \mathbb{R}, \alpha$ is weakly Chaitin T-random if and only if $\alpha$ is Martin-Löf Trandom.

Definition 6 ( $\boldsymbol{T}$-compressibility). Let $T \in \mathbb{R}$ with $T \geq 0$. For any $\alpha \in \mathbb{R}$, we say that $\alpha$ is T-compressible if $H\left(\left.\alpha\right|_{n}\right) \leq T n+o(n)$, which is equivalent to $\lim \sup _{n \rightarrow \infty} H\left(\left.\alpha\right|_{n}\right) / n \leq T$.

For every $T \in[0,1]$ and every $\alpha \in \mathbb{R}$, if $\alpha$ is weakly Chaitin $T$-random and $T$-compressible, then

$$
\lim _{n \rightarrow \infty} \frac{H\left(\alpha \uparrow_{n}\right)}{n}=T
$$

The left-hand side of (1) is referred to as the compression rate of a real $\alpha$ in general. Note, however, that (11) does not necessarily imply that $\alpha$ is weakly Chaitin $T$-random. Thus, the notion of partial randomness is a stronger representation of compression rate.

In the works [1819, we generalized Chaitin $\Omega$ number to $\Omega(T)$ as follows. For each optimal computer $V$ and each real $T>0$, the generalized halting probability $\Omega_{V}(T)$ of $V$ is defined by

$$
\Omega_{V}(T)=\sum_{p \in \operatorname{dom} V} 2^{-\frac{|p|}{T}}
$$

Thus, $\Omega_{V}(1)=\Omega_{V}$. If $0<T \leq 1$, then $\Omega_{V}(T)$ converges and $0<\Omega_{V}(T)<1$, since $\Omega_{V}(T) \leq \Omega_{V}<1$. The following theorem holds for $\Omega_{V}(T)$.

Theorem 4 (Tadaki $[\mathbf{1 8}, 19]$ ). Let $V$ be an optimal computer and let $T \in \mathbb{R}$.

(i) If $0<T \leq 1$ and $T$ is computable, then $\Omega_{V}(T)$ is weakly Chaitin $T$-random and $T$-compressible.

(ii) If $1<T$, then $\Omega_{V}(T)$ diverges to $\infty$.

\section{Previous results on the randomness of an r.e. real}

In this section, we review the previous results on the randomness of an r.e. real. First we review some notions on r.e. reals.

Definition 7 ( $\Omega$-likeness). For any r.e. reals $\alpha$ and $\beta$, we say that $\alpha$ dominates $\beta$ if there are computable, increasing sequences $\left\{a_{n}\right\}$ and $\left\{b_{n}\right\}$ of rationals and $c \in \mathbb{N}^{+}$such that $\lim _{n \rightarrow \infty} a_{n}=\alpha, \lim _{n \rightarrow \infty} b_{n}=\beta$, and $c\left(\alpha-a_{n}\right) \geq \beta-b_{n}$ for all $n \in \mathbb{N}$. An r.e. real $\alpha$ is called $\Omega$-like if it dominates all r.e. reals.

Solovay [15] showed the following theorem. For its proof, see also Theorem 4.9 of [1]. 
Theorem 5 (Solovay [15]). For every r.e. reals $\alpha$ and $\beta$, if $\alpha$ dominates $\beta$ then $H\left(\beta \uparrow_{n}\right) \leq H\left(\alpha \uparrow_{n}\right)+O(1)$ for all $n \in \mathbb{N}^{+}$.

Definition 8 (universality). A computable, increasing and converging sequence $\left\{a_{n}\right\}$ of rationals is called universal if for every computable, increasing and converging sequence $\left\{b_{n}\right\}$ of rationals there exists $c \in \mathbb{N}^{+}$such that $c\left(\alpha-a_{n}\right) \geq \beta-b_{n}$ for all $n \in \mathbb{N}$, where $\alpha=\lim _{n \rightarrow \infty} a_{n}$ and $\beta=\lim _{n \rightarrow \infty} b_{n}$.

The previous results on the equivalent characterizations of randomness for an r.e. real are summarized in the following theorem.

Theorem $6([\mathbf{1 4} 4, \mathbf{1 5}, \mathbf{1}, \mathbf{7}, \mathbf{2 0}])$. Let $\alpha$ be an r.e. real with $0<\alpha<1$. Then the following conditions are equivalent:

(i) The real $\alpha$ is weakly Chaitin random.

(ii) The real $\alpha$ is Martin-Löf random.

(iii) The real $\alpha$ is $\Omega$-like.

(iv) For every r.e. real $\beta, H\left(\left.\beta\right|_{n}\right) \leq H\left(\left.\alpha\right|_{n}\right)+O(1)$ for all $n \in \mathbb{N}^{+}$.

(v) There exists an optimal computer $V$ such that $\alpha=\Omega_{V}$.

(vi) There exists a universal probability $m$ such that $\alpha=\sum_{s \in\{0,1\}^{*}} m(s)$.

(vii) Every computable, increasing sequence of rationals which converges to $\alpha$ is universal.

(viii) There exists a universal computable, increasing sequence of rationals which converges to $\alpha$.

The historical remark on the proofs of equivalences in Theorem 6 is as follows. Schnorr 14 showed that (i) and (ii) are equivalent to each other. Chaitin 4] showed that (v) implies (i). Solovay [15] showed that (v) implies (iii), (iii) implies (iv), and (iii) implies (i). Calude, Hertling, Khoussainov, and Wang 11 showed that (iii) implies (v), and (v) implies (vii). Kučera and Slaman [7] showed that (ii) implies (vii). Finally, (vi) was inserted in the course of the derivation from (v) to (viii) by Tadaki [20].

\section{New results on the partial randomness of an r.e. real}

In this section, we generalize Theorem 6 above over the notion of partial randomness. For that purpose, we first introduce some new notions. Let $T$ be an arbitrary real with $0<T \leq 1$ throughout the rest of this paper. These notions are parametrized by the real $T 1$

Definition 9 ( $\boldsymbol{T}$-convergence). An increasing sequence $\left\{a_{n}\right\}$ of reals is called $T$-convergent if $\sum_{n=0}^{\infty}\left(a_{n+1}-a_{n}\right)^{T}<\infty$. An r.e. real $\alpha$ is called $T$-convergent if there exists a T-convergent computable, increasing sequence of rationals which converges to $\alpha$, i.e., if there exists an increasing sequence $\left\{a_{n}\right\}$ of rationals such that (i) $\left\{a_{n}\right\}$ is T-convergent, (ii) $\left\{a_{n}\right\}$ is computable, and (iii) $\lim _{n \rightarrow \infty} a_{n}=\alpha$.

\footnotetext{
1 The parameter $T$ corresponds to the notion of "temperature" in the statistical mechanical interpretation of algorithmic information theory developed by Tadaki 2122.
} 
Note that every increasing and converging sequence of reals is 1-convergent, and thus every r.e. real is 1-convergent. In general, based on the following lemma, we can freely switch from " $T$-convergent computable, increasing sequence of reals" to " $T$-convergent computable, increasing sequence of rationals."

Lemma 1. For every $\alpha \in \mathbb{R}, \alpha$ is an r.e. T-convergent real if and only if there exists a T-convergent computable, increasing sequence of reals which converges to $\alpha$.

The following argument illustrates the way of using Lemma 1; Let $V$ be an optimal computer, and let $p_{0}, p_{1}, p_{2}, \ldots$ be a recursive enumeration of the r.e. set $\operatorname{dom} V$. Then $\Omega_{V}(T)=\sum_{i=0}^{\infty} 2^{-\left|p_{i}\right| / T}$, and the increasing sequence $\left\{\sum_{i=0}^{n} 2^{-\left|p_{i}\right| / T}\right\}_{n \in \mathbb{N}}$ of reals is $T$-convergent since $\Omega_{V}=\sum_{i=0}^{\infty} 2^{-\left|p_{i}\right|}<1$. If $T$ is computable, then this sequence of reals is computable. Thus, by Lemma 1 we have Theorem 7 below.

Theorem 7. Let $V$ be an optimal computer. If $T$ is computable, then $\Omega_{V}(T)$ is an r.e. T-convergent real.

Definition $10(\Omega(T)$-likeness). An r.e. real $\alpha$ is called $\Omega(T)$-like if it dominates all r.e. T-convergent reals.

Note that an r.e. real $\alpha$ is $\Omega(1)$-like if and only if $\alpha$ is $\Omega$-like.

Definition 11 ( $T$-universality). A computable, increasing and converging sequence $\left\{a_{n}\right\}$ of rationals is called T-universal if for every $T$-convergent computable, increasing and converging sequence $\left\{b_{n}\right\}$ of rationals there exists $c \in \mathbb{N}^{+}$ such that $c\left(\alpha-a_{n}\right) \geq \beta-b_{n}$ for all $n \in \mathbb{N}$, where $\alpha=\lim _{n \rightarrow \infty} a_{n}$ and $\beta=\lim _{n \rightarrow \infty} b_{n}$.

Note that a computable, increasing and converging sequence $\left\{a_{n}\right\}$ of rationals is 1-universal if and only if $\left\{a_{n}\right\}$ is universal.

Using the notions introduced above, Theorem 6 is generalized as follows.

Theorem 8 (main result). Let $\alpha$ be an r.e. real with $0<\alpha<1$. Suppose that $T$ is computable. Then the following conditions are equivalent:

(i) The real $\alpha$ is weakly Chaitin T-random.

(ii) The real $\alpha$ is Martin-Löf T-random.

(iii) The real $\alpha$ is $\Omega(T)$-like.

(iv) For every r.e. $T$-convergent real $\beta, H\left(\left.\beta\right|_{n}\right) \leq H\left(\left.\alpha\right|_{n}\right)+O(1)$ for all $n \in \mathbb{N}^{+}$.

(v) For every r.e. $T$-convergent real $\gamma>0$, there exist an r.e. real $\beta \geq 0$ and $a$ rational $q>0$ such that $\alpha=\beta+q \gamma$.

(vi) For every optimal computer $V$, there exist an r.e. real $\beta \geq 0$ and a rational $q>0$ such that $\alpha=\beta+q \Omega_{V}(T)$.

(vii) There exist an optimal computer $V$ and an r.e. real $\beta \geq 0$ such that $\alpha=$ $\beta+\Omega_{V}(T)$.

(viii) There exists a universal probability $m$ such that $\alpha=\sum_{s \in\{0,1\}^{*}} m(s)^{\frac{1}{T}}$. 
(ix) Every computable, increasing sequence of rationals which converges to $\alpha$ is T-universal.

(x) There exists a T-universal computable, increasing sequence of rationals which converges to $\alpha$.

We see that Theorem 8 is a massive expansion of Theorem 3 in the case where the real $\alpha$ is r.e. with $0<\alpha<1$. The condition (vii) of Theorem 8 corresponds to the condition (v) of Theorem 6. Note, however, that, in the condition (vii) of Theorem 8 , a non-negative r.e. real $\beta$ is needed. The reason is as follows: In the case of $\beta=0$, the possibility that $\alpha$ is weakly Chaitin $T^{\prime}$-random with a real $T^{\prime}>T$ is excluded by the $T$-compressibility of $\Omega_{V}(T)$ imposed by Theorem 4 (i). However, this exclusion is inconsistent with the condition (i) of Theorem 8 .

Theorem 8 can be proved by generalizing the proof of Theorem 6 over the notion of partial randomness. For example, using Lemma 2 below, the implication (ii) $\Rightarrow(v)$ of Theorem 8 is proved as follows, in which the notion of $T$-convergence plays an important role.

Proof (of (ii) $\Rightarrow$ (v) of Theorem 8). Suppose that $\gamma$ is an arbitrary r.e. Tconvergent real with $\gamma>0$. Then there exists a $T$-convergent computable, increasing sequence $\left\{c_{n}\right\}$ of rationals which converges to $\gamma$. Since $\gamma>0$, without loss of generality we can assume that $c_{0}=0$. We choose any one rational $\varepsilon>0$ such that $\sum_{n=0}^{\infty}\left[\varepsilon\left(c_{n+1}-c_{n}\right)\right]^{T} \leq 1$. Such $\varepsilon$ exists since the sequence $\left\{c_{n}\right\}$ is $T$-convergent. Note that the sequence $\left\{\varepsilon\left(c_{n+1}-c_{n}\right)\right\}$ is a computable sequence of positive rationals. Thus, since $\alpha$ is a positive r.e. real and also Martin-Löf $T$-random by the assumption, it follows from Lemma 2 below that there exist a computable, increasing sequence $\left\{a_{n}\right\}$ of rationals and a rational $r>0$ such that $a_{n+1}-a_{n}>r \varepsilon\left(c_{n+1}-c_{n}\right)$ for every $n \in \mathbb{N}, a_{0}>0$, and $\alpha=\lim _{n \rightarrow \infty} a_{n}$. We then define a sequence $\left\{b_{n}\right\}$ of positive rationals by $b_{n}=a_{n+1}-a_{n}-r \varepsilon\left(c_{n+1}-c_{n}\right)$. It follows that $\left\{b_{n}\right\}$ is a computable sequence of rationals and $\sum_{n=0}^{\infty} b_{n}$ converges to $\alpha-a_{0}-r \varepsilon\left(\gamma-c_{0}\right)$. Thus we have $\alpha=a_{0}+\sum_{n=0}^{\infty} b_{n}+r \varepsilon \gamma$, where $a_{0}+\sum_{n=0}^{\infty} b_{n}$ is a positive r.e. real. This completes the proof.

Lemma 2. Let $\alpha$ be an r.e. real, and let $\left\{d_{n}\right\}$ be a computable sequence of positive rationals such that $\sum_{n=0}^{\infty} d_{n}{ }^{T} \leq 1$. If $\alpha$ is Martin-Löf T-random, then for every $\varepsilon>0$ there exist a computable, increasing sequence $\left\{a_{n}\right\}$ of rationals and a rational $q>0$ such that $a_{n+1}-a_{n}>q d_{n}$ for every $n \in \mathbb{N}, a_{0}>\alpha-\varepsilon$, and $\alpha=\lim _{n \rightarrow \infty} a_{n}$.

Lemma 2 can be proved, based on the generalization of the techniques used in the proof of Theorem 2.1 of Kučera and Slaman 7 over partial randomness. In addition to the proof of Lemma 2 the complete proof of Theorem 8 will be described in a full version of this paper, which is in preparation.

Theorem 8 has many important applications. One of the main applications is to give many characterizations of the dimension of an individual r.e. real, some of which will be presented in the next section. As another consequence of Theorem 8, we can obtain Corollary 1 below for example, which follows immediately from the implication (vii) $\Rightarrow$ (iv) of Theorem 8 and Theorem 7 
Corollary 1. Suppose that $T$ is computable. Then, for every two optimal computers $V$ and $W, H\left(\left.\Omega_{V}(T)\right|_{n}\right)=H\left(\left.\Omega_{W}(T)\right|_{n}\right)+O(1)$ for all $n \in \mathbb{N}^{+}$.

Note that the computability of $T$ is important for Theorem 8 to hold. For example, we cannot allow $T$ to be simply an r.e. real in Theorem 8 ,

The notion of $T$-convergence has many interesting properties, in addition to the properties which we saw above. In Section 6, we investigate further properties of the notion of $T$-convergence.

\section{New characterizations of the dimension of an r.e. real}

In this section we apply Theorem 8 to give many characterizations of dimension for an individual r.e. real. In the works [1819, we introduced the notions of six "algorithmic dimensions", 1st, 2nd, 3rd, 4th, upper, and lower algorithmic dimensions as fractal dimensions for a subset $F$ of $N$-dimensional Euclidean space $\mathbb{R}^{N}$. These notions are defined based on the notion of partial randomness and compression rate by means of program-size complexity. We then showed that all the six algorithmic dimensions equal to the Hausdorff dimension for any self-similar set which is computable in a certain sense. The class of such selfsimilar sets includes familiar fractal sets such as the Cantor set, von Koch curve, and Sierpiński gasket. In particular, the notion of lower algorithmic dimension for a subset $F$ of $\mathbb{R}$ is defined as follows.

Definition 12 (lower algorithmic dimension, Tadaki [19]). Let $F$ be a nonempty subset of $\mathbb{R}$. The lower algorithmic dimension $\underline{\operatorname{dim}}_{A} F$ of $F$ is defined by $\underline{\operatorname{dim}}_{A} F=\sup _{x \in F} \liminf _{n \rightarrow \infty} H\left(x \Gamma_{n}\right) / n$.

Thus, for every $\alpha \in \mathbb{R}$,

$$
\underline{\operatorname{dim}}_{A}\{\alpha\}=\liminf _{n \rightarrow \infty} \frac{H\left(\alpha \uparrow_{n}\right)}{n} .
$$

Independently of us, Lutz [8] introduced the notion of constructive dimension of an individual real $\alpha$ using the notion of lower semicomputable $s$-supergale with $s \in[0, \infty)$, and then Mayordomo [10] showed that, for every real $\alpha$, the constructive dimension of $\alpha$ equals to the right-hand side of (2). Thus, the constructive dimension of $\alpha$ is precisely the lower algorithmic dimension $\underline{\operatorname{dim}}_{A}\{\alpha\}$ of $\alpha$ for every real $\alpha$.

Using Lemma 3 below, we can convert each of all the conditions in Theorem 8 into a characterization of the lower algorithmic dimension $\underline{\operatorname{dim}}_{A}\{\alpha\}$ for any r.e. real $\alpha$.

Lemma 3. Let $\alpha \in \mathbb{R}$. For every $t \in[0, \infty), \alpha$ is weakly Chaitin $t$-random if $t<\underline{\operatorname{dim}}_{A}\{\alpha\}$, and $\alpha$ is not weakly Chaitin t-random if $t>\underline{\operatorname{dim}}_{A}\{\alpha\}$.

Proof. Let $\alpha \in \mathbb{R}$, and let $t \in[0, \infty)$. Assume first that $t<\underline{\operatorname{dim}}_{A}\{\alpha\}$. Then, since $\underline{\operatorname{dim}}_{A}\{\alpha\} n \leq H\left(\left.\alpha\right|_{n}\right)+o(n)$ for all $n \in \mathbb{N}^{+}$, we see that

$$
t n+\left(\underline{\operatorname{dim}}_{A}\{\alpha\}-t-\frac{o(n)}{n}\right) n \leq \underline{\operatorname{dim}}_{A}\{\alpha\} n-o(n) \leq H\left(\left.\alpha\right|_{n}\right)
$$


for all $n \in \mathbb{N}^{+}$. Thus, since $\underline{\operatorname{dim}}_{A}\{\alpha\}-t-o(n) / n>0$ for all sufficiently large $n$, we see that $\alpha$ is weakly Chaitin $t$-random.

On the other hand, assume that $\alpha$ is weakly Chaitin $t$-random. Then we see that $t \leq \liminf _{n \rightarrow \infty} H\left(\left.\alpha\right|_{n}\right) / n=\underline{\operatorname{dim}}_{A}\{\alpha\}$. Thus, if $t>\underline{\operatorname{dim}}_{A}\{\alpha\}$ then $\alpha$ is not weakly Chaitin $t$-random. This completes the proof.

For example, using Lemma 3 the condition (iii) in Theorem 8 is converted as follows. In this paper, we interpret the supremum $\sup \emptyset$ of the empty set as 0 .

Theorem 9. Let $\alpha$ be an r.e. real. Then, for every $t \in(0,1], \alpha$ is $\Omega(t)$-like if $t<\underline{\operatorname{dim}}_{A}\{\alpha\}$, and $\alpha$ is not $\Omega(t)$-like if $t>\underline{\operatorname{dim}}_{A}\{\alpha\}$. Thus,

$$
\underline{\operatorname{dim}}_{A}\{\alpha\}=\sup \{t \in(0,1] \mid \alpha \text { is } \Omega(t) \text {-like }\} .
$$

On the other hand, the condition (viii) in Theorem 8 is converted as follows, using Lemma 3. Here $\mathbb{R}_{c}$ denotes the set of all computable reals.

Theorem 10. Let $\alpha$ be an r.e. real with $0<\alpha<1$, Then, for every $t \in(0,1] \cap$ $\mathbb{R}_{c}$, if $t<\underline{\operatorname{dim}}_{A}\{\alpha\}$ then $\alpha=\sum_{s \in\{0,1\}^{*}} m(s)^{\frac{1}{t}}$ for some universal probability $m$, and if $t>\underline{\operatorname{dim}}_{A}\{\alpha\}$ then $\alpha \neq \sum_{s \in\{0,1\}^{*}} m(s)^{\frac{1}{t}}$ for any universal probability m. Thus, $\underline{\operatorname{dim}}_{A}\{\alpha\}=\sup S$, where $S$ is the set of all $t \in(0,1] \cap \mathbb{R}_{c}$ such that $\alpha=\sum_{s \in\{0,1\}^{*}} m(s)^{\frac{1}{t}}$ for some universal probability $m$.

In the same manner, using Lemma 3 we can convert each of the remaining eight conditions in Theorem 8 also into a characterization of the lower algorithmic dimension of an r.e. real. In a full version of this paper, we will describe the complete list of the ten characterizations of the lower algorithmic dimension obtained from Theorem 8

\section{Further properties of $\boldsymbol{T}$-convergence}

In this section, we investigate further properties of the notion of $T$-convergence. First, as one of the applications of Theorem 8 the following theorem can be obtained.

Theorem 11. Suppose that $T$ is computable. For every r.e. real $\alpha$, if $\alpha$ is $T$ convergent, then $\alpha$ is T-compressible.

Proof. Using (vii) $\Rightarrow$ (iv) of Theorem 8 , we see that, for every r.e. $T$-convergent real $\alpha, H\left(\left.\alpha\right|_{n}\right) \leq H\left(\left.\Omega_{U}(T)\right|_{n}\right)+O(1)$ for all $n \in \mathbb{N}^{+}$. It follows from Theorem 4 (i) that $\alpha$ is $T$-compressible for every r.e. $T$-convergent real $\alpha$.

In the case of $T<1$, the converse of Theorem 11 does not hold, as seen in Theorem 12 below in a sharper form. Theorem 12 can be proved partly using (vii) $\Rightarrow$ (ix) of Theorem 8 .

Theorem 12. Suppose that $T$ is computable and $T<1$. Then there exists an r.e. real $\eta$ such that (i) $\eta$ is weakly Chaitin $T$-random and T-compressible, and (ii) $\eta$ is not $T$-convergent. 
Let $T_{1}$ and $T_{2}$ be arbitrary computable reals with $0<T_{1}<T_{2}<1$, and let $V$ be an arbitrary optimal computer. By Theorem 4 (i) and Theorem 11 , we see that the r.e. real $\Omega_{V}\left(T_{2}\right)$ is not $T_{1}$-convergent and therefore every computable, increasing sequence $\left\{a_{n}\right\}$ of rationals which converges to $\Omega_{V}\left(T_{2}\right)$ is not $T_{1}$-convergent. At this point, conversely, the following question arises naturally: Is there any computable, increasing sequence of rationals which converges to $\Omega_{V}\left(T_{1}\right)$ and which is not $T_{2}$-convergent ? We can answer this question affirmatively in the form of Theorem 13 below.

Theorem 13. Let $T_{1}$ and $T_{2}$ be arbitrary computable reals with $0<T_{1}<T_{2}<$ 1. Then there exist an optimal computer $V$ and a computable, increasing sequence $\left\{a_{n}\right\}$ of rationals such that (i) $\Omega_{V}\left(T_{1}\right)=\lim _{n \rightarrow \infty} a_{n}$, (ii) $\left\{a_{n}\right\}$ is T-convergent for every $T \in\left(T_{2}, \infty\right)$, and (iii) $\left\{a_{n}\right\}$ is not $T$-convergent for every $T \in\left(0, T_{2}\right]$.

\section{Concluding remarks}

In this paper, we have generalized the equivalent characterizations of randomness of a recursively enumerable real over the notion of partial randomness, so that the generalized characterizations are all equivalent to the weak Chaitin $T$-randomness. As a stronger notion of partial randomness of a real $\alpha$, Tadaki 1819 introduced the notion of the Chaitin $T$-randomness of $\alpha$, which is defined as the condition on $\alpha$ that $\lim _{n \rightarrow \infty} H\left(\left.\alpha\right|_{n}\right)-T n=\infty 2$ Thus, future work may aim at modifying our equivalent characterizations of partial randomness so that they become equivalent to the Chaitin $T$-randomness.

Acknowledgments. This work was supported by KAKENHI, Grant-in-Aid for Scientific Research (C) (20540134), by SCOPE from the Ministry of Internal Affairs and Communications of Japan, and by CREST from Japan Science and Technology Agency.

\section{References}

1. C. S. Calude, P. H. Hertling, B. Khoussainov, and Y. Wang, "Recursively enumerable reals and Chaitin $\Omega$ numbers," Theoret. Comput. Sci, vol. 255, pp. 125-149, 2001.

2. C. S. Calude, L. Staiger, and S. A. Terwijn, "On partial randomness," Annals of Pure and Applied Logic, vol. 138, pp. 20-30, 2006.

3. C. S. Calude and M. A. Stay, "Natural halting probabilities, partial randomness, and zeta functions," Inform. and Comput., vol. 204, pp. 1718-1739, 2006.

4. G. J. Chaitin, "A theory of program size formally identical to information theory," J. Assoc. Comput. Mach., vol. 22, pp. 329-340, 1975.

5. G. J. Chaitin, Algorithmic Information Theory. Cambridge University Press, Cambridge, 1987.

2 The actual separation of the Chaitin $T$-randomness from the weak Chaitin $T$ randomness is done by Reimann and Stephan [1]. 
6. R. G. Downey and J. Reimann (2007) Algorithmic randomness. Scholarpedia, 2(10):2574, revision \#37278. Available at URL: http://www.scholarpedia.org/article/Algorithmic_randomness

7. A. Kučera and T. A. Slaman, "Randomness and recursive enumerability," SIAM J. Comput., vol. 31, No. 1, pp. 199-211, 2001.

8. J. H. Lutz, Gales and the constructive dimension of individual sequences. Proceedings of the 27th Colloquium on Automata, Languages and Programming (ICALP 2000), Lecture Notes in Computer Science, Springer-Verlag, vol. 1853, pp. 902-913, 2000.

9. P. Martin-Löf, "The definition of random sequences," Information and Control, vol. 9, pp. 602-619, 1966.

10. E. Mayordomo, "A Kolmogorov complexity characterization of constructive Hausdorff dimension," Inform. Process. Lett., vol. 84, pp. 1-3, October 2002.

11. J. Reimann and F. Stephan, On hierarchies of randomness tests. Proceedings of the 9th Asian Logic Conference, World Scientific Publishing, August 16-19, 2005, Novosibirsk, Russia.

12. B. Ya. Ryabko, "Coding of combinatorial sources and Hausdorff dimension." Soviet Math. Dokl., vol. 30, pp. 219-222, 1984.

13. B. Ya. Ryabko, "Noiseless coding of combinatorial sources, Hausdorff dimension, and Kolmogorov complexity," Problems Inform. Transmission, vol. 22, pp. 170$179,1986$.

14. C.-P. Schnorr, "Process complexity and effective random tests," J. Comput. System Sci., vol. 7, pp. 376-388, 1973.

15. R. M. Solovay, "Draft of a paper (or series of papers) on Chaitin's work ... done for the most part during the period of Sept.-Dec. 1974," unpublished manuscript, IBM Thomas J. Watson Research Center, Yorktown Heights, New York, May 1975, 215 pp.

16. L. Staiger, "Kolmogorov complexity and Hausdorff dimension," Inform. and Comput., vol. 103, pp. 159-194, 1993.

17. L. Staiger, "A tight upper bound on Kolmogorov complexity and uniformly optimal prediction," Theory Comput. Systems, vol. 31, pp. 215-229, 1998.

18. K. Tadaki, Algorithmic information theory and fractal sets. Proceedings of 1999 Workshop on Information-Based Induction Sciences (IBIS'99), pp. 105-110, August 26-27, 1999, Syuzenji, Shizuoka, Japan. In Japanese.

19. K. Tadaki, "A generalization of Chaitin's halting probability $\Omega$ and halting selfsimilar sets," Hokkaido Math. J., vol. 31, pp. 219-253, February 2002.

20. K. Tadaki, "An extension of Chaitin's halting probability $\Omega$ to a measurement operator in an infinite dimensional quantum system," Math. Log. Quart., vol. 52, pp. 419-438, 2006.

21. K. Tadaki, A statistical mechanical interpretation of algorithmic information theory. Local Proceedings of Computability in Europe 2008 (CiE 2008), pp. 425-434, June 15-20, 2008, University of Athens, Greece. Extended and Electronic Version Available: http://arxiv.org/abs/0801.4194v1

22. K. Tadaki, Fixed point theorems on partial randomness. Proceedings of the Symposium on Logical Foundations of Computer Science 2009 (LFCS'09), Lecture Notes in Computer Science, Springer-Verlag, vol. 5407, pp. 422-440, January 3-6, 2009.

23. K. Weihrauch, Computable Analysis. Springer-Verlag, Berlin, 2000.

24. A. K. Zvonkin and L. A. Levin, "The complexity of finite objects and the development of the concepts of information and randomness by means of the theory of algorithms," Russian Math. Surveys, vol. 25, no. 6, pp. 83-124, 1970. 